\title{
Predictability of the monthly North Atlantic Oscillation index based on fractal analyses and dynamic system theory
}

\author{
M. D. Martínez ${ }^{1}$, X. Lana ${ }^{2}$, A. Burgueño ${ }^{3}$, and C. Serra ${ }^{2}$ \\ ${ }^{1}$ Dept. de Física Aplicada, Universitat Politècnica de Catalunya, Av. Diagonal 649, 08028 Barcelona, Spain \\ ${ }^{2}$ Dept. de Física i Enginyeria Nuclear, Universitat Politècnica de Catalunya, Av. Diagonal 647, 08028 Barcelona, Spain \\ ${ }^{3}$ Dept. d'Astronomia i Meteorologia, Universitat de Barcelona, C./ Martí Franquès 1, 08028 Barcelona, Spain
}

Received: 25 February 2009 - Revised: 5 February 2010 - Accepted: 20 February 2010 - Published: 5 March 2010

\begin{abstract}
The predictability of the monthly North Atlantic Oscillation, NAO, index is analysed from the point of view of different fractal concepts and dynamic system theory such as lacunarity, rescaled analysis (Hurst exponent) and reconstruction theorem (embedding and correlation dimensions, Kolmogorov entropy and Lyapunov exponents). The main results point out evident signs of randomness and the necessity of stochastic models to represent time evolution of the NAO index. The results also show that the monthly NAO index behaves as a white-noise Gaussian process. The high minimum number of nonlinear equations needed to describe the physical process governing the NAO index fluctuations is evidence of its complexity. A notable predictive instability is indicated by the positive Lyapunov exponents. Besides corroborating the complex time behaviour of the NAO index, present results suggest that random Cantor sets would be an interesting tool to model lacunarity and time evolution of the NAO index.
\end{abstract}

\section{Introduction}

The North Atlantic Oscillation, NAO, index can be defined as the difference between the normalized sea level atmospheric pressures at Gibraltar and South-West Iceland. More specifically, pressures at every location are normalised by subtracting the mean and dividing by the standard deviation. A standard period (1951-1980), instead of the whole recording period, is considered for estimating the mean and the standard deviation (Jones et al., 1997). Monthly and annual NAO indices since 1823 are available at the Climate Research Unit of the University of East Anglia, UK (http://www.cru.uea.ac.uk).

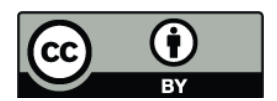

Correspondence to: M. D. Martínez (dolors.martinez@upc.edu)
Figure 1a depicts the time evolution of the monthly NAO index along the period 1825-2007 (2196 monthly records). Instead of monthly NAO indices, running averages of 13month window are represented. Basic statistics of the monthly series are characterized by a range of variation from -6.05 to 6.66 , an expected value of 0.09 and a standard deviation of 1.76. The skewness and kurtosis are very close to zero (0.06 and -0.15 , respectively). In addition, remembering that Muñoz-Díaz and Rodrigo (2004) proved that winter NAO indices are normal distributed, it should not be discarded that monthly NAO indices, without distinguishing seasons, were also normal distributed or, at least, very symmetrically distributed around zero. This hypothesis of normal distribution is verified by the Kolmogorov-Smirnov and D'Agostino K-squared and Jarque-Bera tests (Bera and Jarque, 1981; D'Agostino et al., 1990). The KolmogorovSmirnov statistic for the empirical standardised data is 0.014 . Considering that the width of the Kolmogorov-Smirnov 95\% confidence bands is very approximately given by $1.36 / \sqrt{N}$, with $N$ the number of samples, a critical value of 0.029 is obtained. Thus, given that the statistic for the empirical standardised data does not exceed this critical value, monthly NAO is assumed to be normal distributed. The other two tests, based on samples of skewness and kurtosis and the assumption that both statistics follow a $\chi^{2}$ distribution with two degrees of freedom, also suggest that monthly NAO indices follow a Gaussian distribution. Figure $1 \mathrm{~b}$ compares the empirical and the $N(0,1)$ complementary cumulative distributions. It is observed that the empirical distribution is within the Kolmogorov-Smirnov 95\% confidence bands for all the monthly NAO range.

Nowadays, it is well established that fluctuations of the NAO index are closely related to changes on the pluviometric and temperature regimes at regional and local scales for wide areas of Western Europe, being detected short-term periodic variations ( $2-3$ years) and statistically significant time trends (Ulbrich and Christoph, 1999; Hurrell et al., 2001;

Published by Copernicus Publications on behalf of the European Geosciences Union and the American Geophysical Union. 


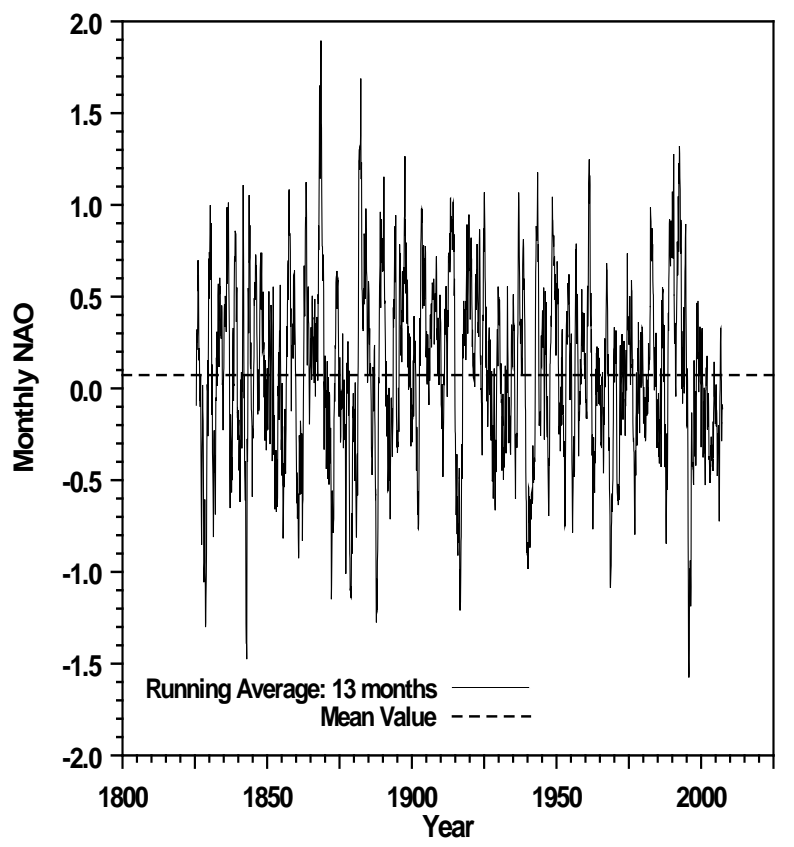

Fig. 1a. Running average of monthly NAO indices for the recording period 1825-2007. Dashed line depicts the mean monthly NAO index for the whole recording period.

Trigo et al., 2002; Martín et al., 2004; Krichak and Alpert, 2005). See Marshall et al. (2001) for a complete review of the NAO variability, including phenomenology, impacts and mechanisms due to ocean-atmosphere interactions.

The quantification of monthly or annual NAO predictability should be very interesting as it would permit the uncertainties on winter forecasts at monthly or seasonal scales in Western Europe to be reduced. Many efforts have also been devoted to search for stochastic models representing NAO fluctuations. A few examples can be found in Feldstein (2000), who analysed time scale, power spectra and noise properties; Stephenson et al. (2000) and Mills (2004), who questioned random walk behaviour and proposed alternative models; and Collette and Ausloos (2004), who applied rescaled and detrended fluctuation analyses and proposed fluid dynamics analogies.

The proposed analysis of NAO predictability is based on several aspects of fractal theory, which application usually requires long data series. Although it is well known the particular relevance of NAO index fluctuations in the winter period, in the present work it would not be appropriate to distinguish winter season from every recording year. First, the length of the analysed series would be notably reduced. Second, it is to be expected that the interpretation of predictive mechanisms and predictive instability could be affected by computational artefacts generated by forced discontinuities in NAO indices at the beginning and the end of every winter period.

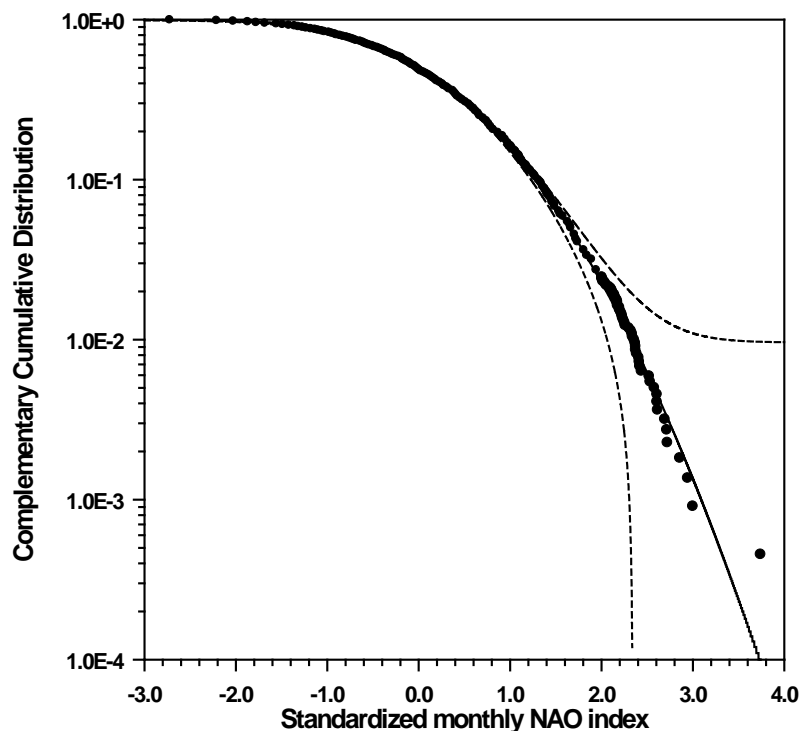

Fig. 1b. Empirical cumulative distribution (dots) of standardised monthly NAO indices fitted to the normal $N(0,1)$ distribution (solid line). Kolmogorov-Smirnov 95\% confidence bands are represented by dashed lines.

The concept of lacunarity (Turcotte, 1997) of monthly NAO indices is analysed and modelled by means of random Cantor sets (Korvin, 1992; Turcotte, 1997). The degree of randomness of NAO index series is quantified by the interpretation of the Hurst exponent of the rescaled analysis (Lomnitz, 1994; Turcotte 1997; Diks, 1999). A deeper insight into the complexity of monthly NAO indices is achieved in terms of the reconstruction theorem (Grassberger and Procaccia, 1983a; Diks, 1999), leading to derive the correlation dimension, interpreted as the minimum number of nonlinear equations governing the physical process of monthly NAO index evolution. The Kolmogorov entropy (Grassberger and Procaccia, 1983b; Cohen and Procaccia, 1983) describes the loss of memory of the physical process, and the Lyapunov exponents (Turcotte, 1997) quantify the predictive instability of consecutive monthly NAO indices.

\section{Lacunarity}

The lacunarity, $L(r)$, with $r$ a segment length of the series analysed, can be interpreted as a measure of the distribution of clusters of consecutive monthly NAO indices exceeding a threshold level $V_{0}$. Consequently, the concept of $L(r)$ would be complementary to that of the cluster dimension (Theiler, 1988). From a quantitative point of view, following Turcotte (1997), $n(s, r)$ is introduced as the number of moving windows of length $r$ (in months) containing segments with a number $s$ of consecutive monthly NAO indices above the threshold $V_{0}$. After that, the probability $p(s, r)$ is evaluated 
as

$p(s, r)=n(s, r) / N(r)$

the total number of possible windows of length $r$ being

$N(r)=\ell-r+1$

with $\ell$ the total number of recording months, including segments and gaps. The first and second order moments of $s$ are

$M_{1}(r)=\sum_{s=1}^{t} s \cdot p(s, r)$

$M_{2}(r)=\sum_{s=1}^{t} s^{2} \cdot p(s, r)$

and finally, the lacunarity is defined as the quotient

$L(r)=M_{2}(r) /\left[M_{1}(r)\right]^{2}$

Details about the computation of $L(r)$ as well as comparisons among L(r)-curves of synthetic series generated from random uniform distributions and pure, random and clumped Cantor sets can be found in Turcotte (1997). Figure 2a shows some examples of L(r)-curves of the NAO index, which are well reproduced by the lacunarity of random Cantor sets. These L(r)-curves are computed for three threshold levels $V_{0}$ equal to $-1.0,0.0$, and 1.0 and related to gap sizes $G$ of the Cantor sets equal to $0.04,0.08$, and 0.15 , respectively. It should be remembered that the fractal dimension of the Cantor set is equal to $\log 2 / \log [(1-G) / 2]$ (Korvin, 1992), given that, for any new iteration of the Cantor set generation, all elements are fragmented into two new pieces and a gap. For the rest of threshold levels, $V_{0}$, at intervals of 0.25 , the fit of the empirical $L(r)$ to the synthetic lacunarity is also quite good and, as expected, $G$ increases systematically with $V_{0}$ and all curves tend asymptotically to 1.0 for $r$ tending to $\infty$. It is worth mentioning that $\mathrm{L}(\mathrm{r})$-curves are not well fitted to pure or clumped Cantor sets, whatever the length $r$ and level $V_{0}$.

A complementary analysis of the lacunarity is achieved by representing the evolution of $L(1)$ with $V_{0}$ in Fig. $2 b$. It is also remarkable that for $r$ equal to 1 , a picture of the monthly behaviour of NAO index is obtained. Similarly to the pluviometric series analysed by Martínez et al. (2007), L(1) can be described by the exponential laws

$$
\begin{aligned}
& L\left(1, V_{0}\right)=1.766 \cdot e^{0.226 \cdot V_{0}} ;-2.25 \leq V_{0}<-0.25 \\
& L\left(1, V_{0}\right)=1.863 \cdot e^{0.627 \cdot V_{0}} ;-0.25 \leq V_{0}<2.25 \\
& L\left(1, V_{0}\right)=0.404 \cdot e^{1.303 \cdot V_{0}} ; \quad 2.25 \leq V_{0} \leq 5.0
\end{aligned}
$$

with square regression coefficients $0.98,0.99$, and 0.99 , respectively. As expected, for threshold values of $V_{0}$ less than $-2.25, L(1)$ tends asymptotically to 1.0 . For threshold levels exceeding 5.0, $L(1)$ depicts a sharp increase up to $V_{0}$ close

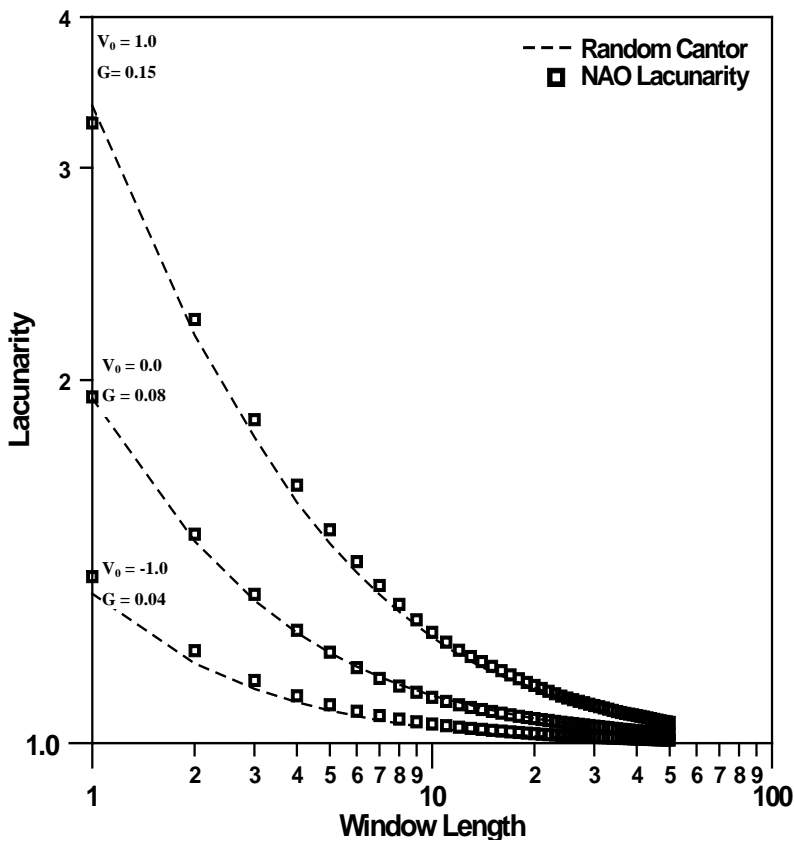

Fig. 2a. Evolution of the empirical lacunarity of monthly NAO index and lacunarity derived from a random Cantor model for three $V_{0}$ values. Window lengths are given in months.

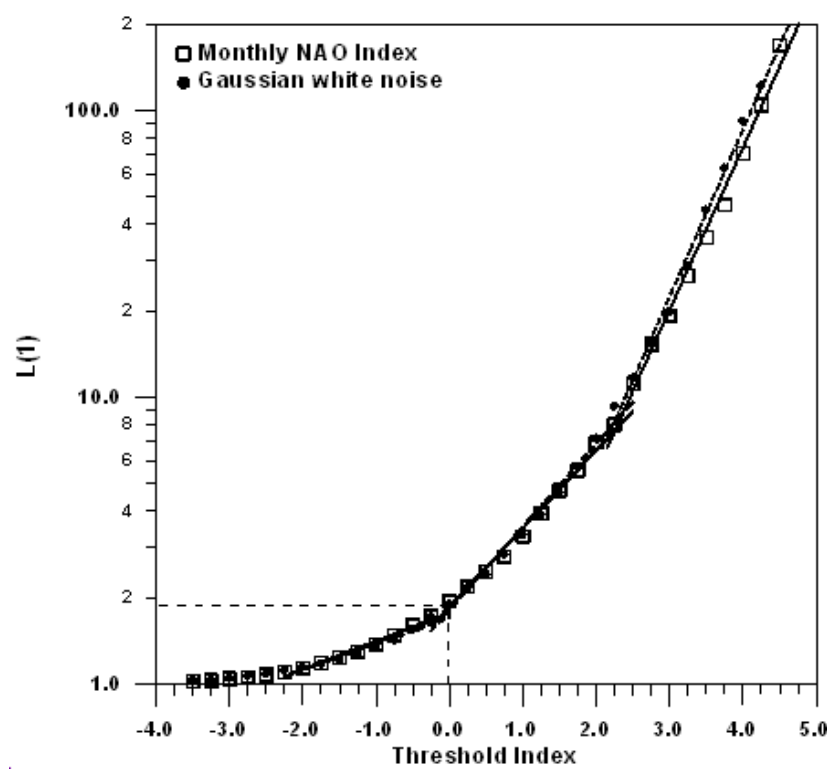

Fig. 2b. Evolution of $L(1)$ for a wide range of threshold values $V_{0}$. Dashed and solid straight lines depict the lacunarity corresponding to Gaussian white-noise and real NAO indices, respectively.

to 6.5 , which is the highest monthly NAO index observed. In agreement with Mandelbrot (1982), lacunarity measures the distribution of gap sizes in the series analysed. Large lacunarity implies large gaps, whereas small lacunarity means a more uniform distribution of smaller gaps. According to Eq. (5) and Fig. 2b, for $r$ equal to 1, monthly NAO indices 
evolve from small gaps quite homogeneously distributed to large gaps, especially for threshold values $V_{0}$ exceeding a critical value of -0.25 . It is also worth mentioning that this evolution towards large gaps is not monotonous, but it is characterised by a fast increase for $V_{0}$ above 2.25 . Additionally, the symmetrical distribution of NAO indices around zero is strongly enhanced by the fact that a threshold $V_{0}=0.05$ generates a lacunarity of 1.99 , very close to the theoretical value of 2.0, which would correspond to a threshold of 0.0 for a perfect symmetrical distribution. This distribution around zero of monthly NAO indices would be in agreement with a Gaussian distribution with mean equal to 0.09 and standard deviation of 1.75 .

Another fact highlights the similarity of the monthly NAO indices and the Gaussian distribution. The behaviour of Gaussian white noise series, with the same number of monthly values, is also represented in Fig. $2 \mathrm{~b}$. The $L(1)$ curves for monthly NAO indices and the Gaussian noise series, generated with the same expected value and standard deviation that NAO indices, are very similar.

\section{Rescaled analysis}

The rescaled analysis can be interpreted as an alternative measure of long-range correlation in time series (Turcotte, 1997). This procedure consists of the computation of mean values, cumulative differences, and after that, maximum range of the integrated signal, $R(\tau)$, and standard deviations, $S(\tau)$, for subsets of series with different number $\tau$ of consecutive elements. If there exists fractal behaviour, the Hurst exponent, $H$, defined as positive, is introduced through the expression

$R(\tau) / S(\tau)=a \tau^{H}$

It should be remembered that a value of $H$ clearly exceeding 0.5 is a sign of time persistence in the dynamic system. Then, time trends deduced for the analysed segment of length $\tau$ will remain and the best prediction should be that based on the extrapolation of these time trends. On the contrary, values of $H$ well below 0.5 indicate antipersistence. Time trends will reverse and the best prediction should be an average over the segment of length $\tau$. Finally, values of $H$ very close to 0.5 indicate randomness. Successive steps of the dynamic system are then uncorrelated and the best prediction should be the last measure. A typical example could be a Gaussian white noise series.

A log-log regression of Eq. (6) applied to annual NAO indices yields for $H$ a value of $0.53\left(\rho^{2} \approx 0.99\right)$. Then, the rescaled analysis shows the random character of the NAO indices at annual scale. The robustness of $H$ is tested repeating the rescaled analysis removing a 5\% of annual NAO indices, first at the beginning of the record, and after at the end. Hurst exponents derived for both tests are very close to that obtained for the whole series and differences are de- tected only in the third decimal digit. In addition, the empirical monthly NAO indices are compared with a Gaussian white noise series with the same length and the same mean and standard deviation as the empirical series. The results obtained are shown in Fig. 3a. Both Hurst exponents are very similar and R/S series are well fitted to the corresponding power laws. R/S analyses of monthly NAO indices by Collette and Ausloos (2004) yields very similar $H$ values. In agreement with the usual interpretation of the Hurst exponent (Turcotte, 1997), persistence $(0.5<H<1.0)$ or anti-persistence $(0<H<0.5)$ have to be discarded in front of randomness $(H \approx 0.5)$ for annual and monthly NAO indices. From this point of view, prediction of NAO indices would be questionable and time trends would not be of great help due to lack of persistence and evident signs of white noise behaviour. Figure $3 \mathrm{~b}$ depicts the evolution of the autocorrelation of the monthly NAO indices and Gaussian white noise for lags up to 100 months. The coefficients are very small, varying within the $(-0.05,0.10)$ range, and are slightly lower for the Gaussian series, in agreement with the lack of correlation detected by the rescaled analysis.

\section{Reconstruction theorem}

The goals of the reconstruction theorem are to determine the minimum number of nonlinear equations governing a physical process, the loss of memory of this process from its beginning up to some present state and its predictive instability. The first two scopes are achieved from the correlation integral, $C(r)$, and the third from the Lyapunov exponents.

Let's $\left\{X_{j}\right\}(j=1, \ldots, N)$ be the series of monthly NAO indices and

$\boldsymbol{Z}_{k}=\left(X_{k+1}, \ldots, X_{k+m-1}, X_{k+m}\right)$

a m-dimensional vector representing a reconstruction of the physical process in a m-dimensional space. The correlation integral $C(r)$ can be computed then as

$C(r)=\lim _{N \rightarrow \infty} \frac{1}{N^{2}} \sum_{i, j}^{N} H\left\{r-\left|Z_{i}-Z_{j}\right|\right\}$

with $H\{\cdot\}$ the Heaviside function. Equation (8) should be interpreted as the number of points in the m-dimensional space within an m-sphere of radius $r . C(r)$ behaves as (Diks, 1999)

$C(r)=A_{m} r^{\mu(m)} e^{-m \kappa}$

$\mu(m)$ being the correlation dimension and $\kappa$ the Kolmogorov entropy. The stationary value of $\mu(m), \mu^{*}$, for a high enough dimension $m$ (embedding dimension $d_{\mathrm{E}}$ ) establishes the minimum number of nonlinear equations governing the physical process and $\kappa$ quantifies its loss of memory.

Although the evolution of $C(r)$ departs from Eq. (9a) at low values of $r$ due to the effects of lacunarity, and $C(r)$ saturates at 1.0 for high values of $r$, a linear regression of 


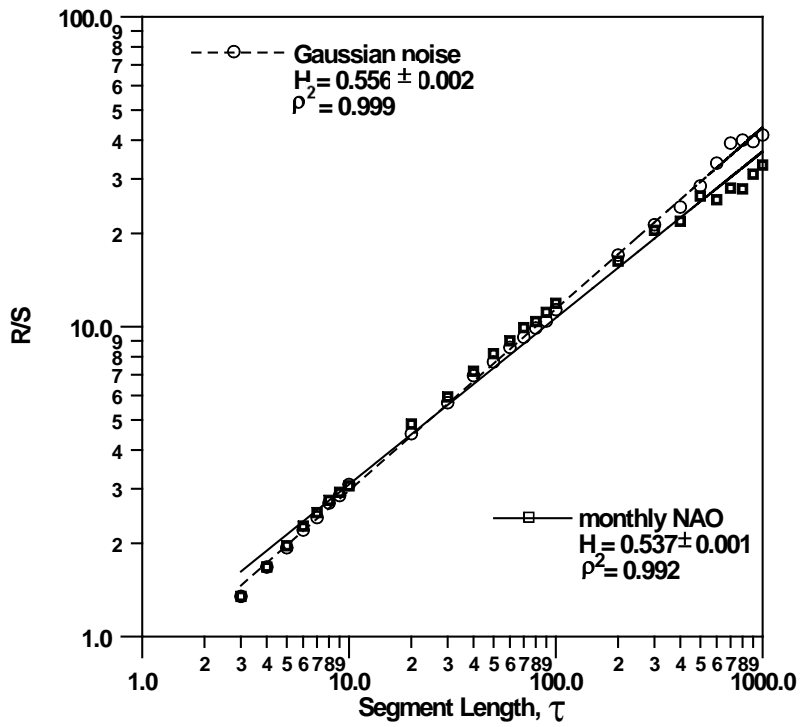

Fig. 3a. Rescaled analysis for the monthly NAO and Gaussian white-noise series.

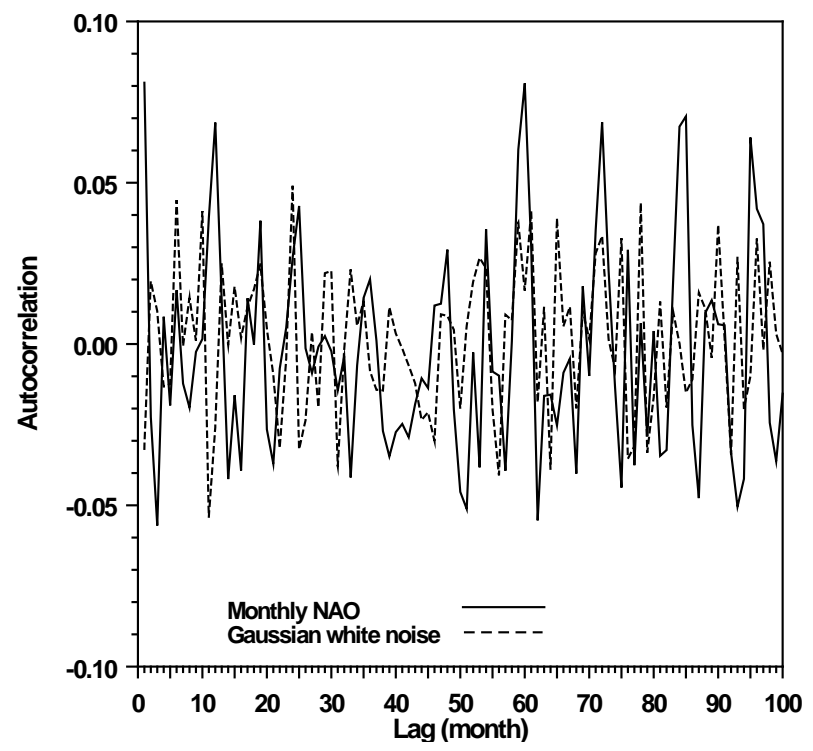

Fig. 3b. Autocorrelation coefficients for lags up to 100 months.

$\log _{\mathrm{e}}\{C(r)\}$ with respect to $\log _{\mathrm{e}}(r)$ for the appropriate range of $r$ permits a good estimation of $\mu(m)$ when increasing dimension $m$. Additionally, by taking logarithms in Eq. (9a)

$\log _{\mathrm{e}}\{C(r)\}=\log _{\mathrm{e}}\left\{A_{m}\right\}-m \kappa+\mu(m) \log _{\mathrm{e}}\{r\}=\alpha(m)+$

$+\mu(m) \log _{\mathrm{e}}\{r\}$

and $\alpha(m)=\log _{\mathrm{e}}\left(A_{m}\right)-m \kappa$ permits to obtain the Kolmogorov entropy, provided that $A_{m+1} \approx A_{m}$ for $m$ tending to $\infty$. The $\log -\log$ evolution of $C(r)$ with m for monthly NAO indices is depicted in Fig. 4a. It is observed that the range of $r$ decreases when increasing $m$, and the effects of lacunarity and saturation are always observed at the beginning and the

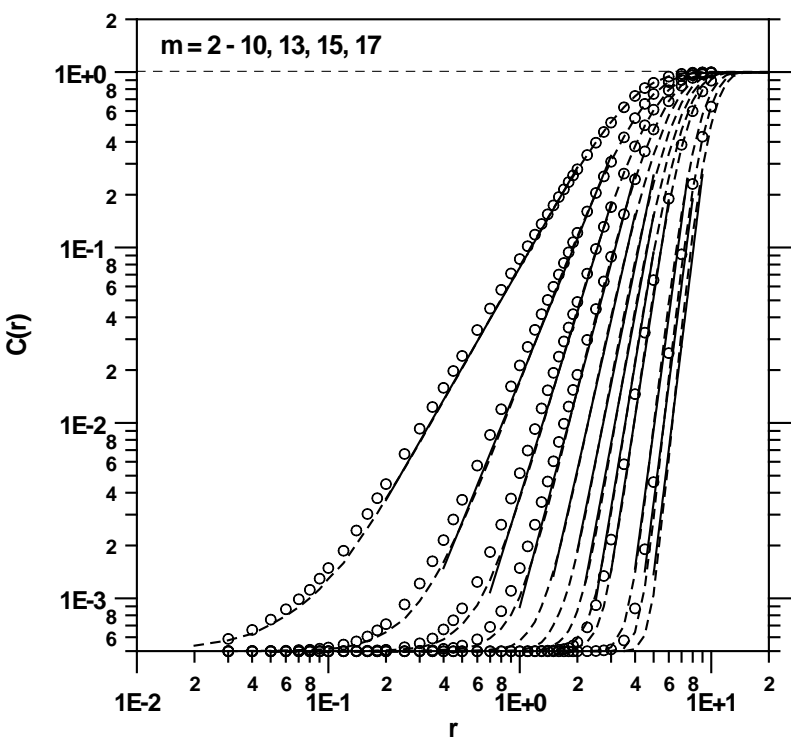

Fig. 4a. Correlation integral, $C(r)$, of the monthly NAO indices (dashed lines) for several reconstruction dimensions $m$ ranging from 2 to 17 , compared with a Gaussian white-noise series (open circles) for $m$ equal to $2,3,4,5,10$, and 15 . Solid straight lines represent the $\log -\log$ evolution of $C(r)$.

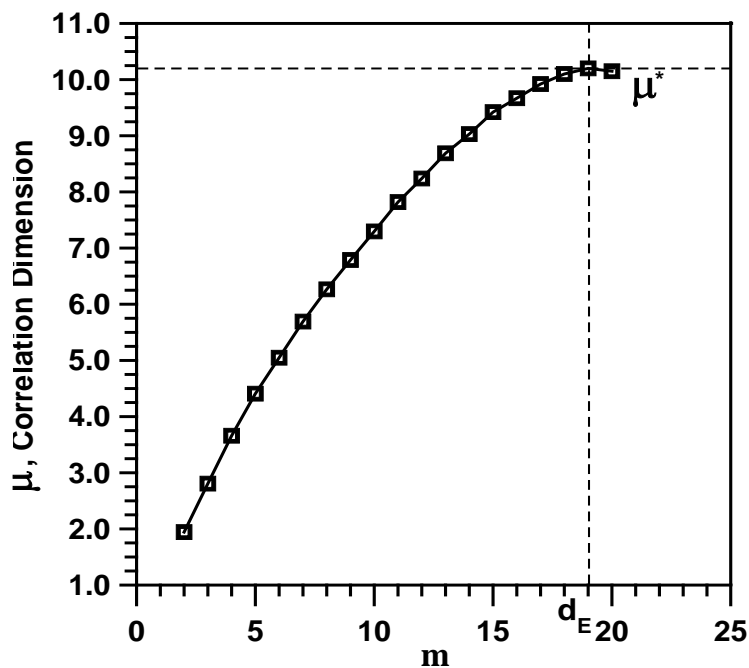

Fig. 4b. Evolution of the correlation dimension with $m$.

end of $C(r)$, respectively. The evolution of $C(r)$ with $r$ for the monthly NAO indices and the Gaussian white noise series at several reconstruction dimensions $m(2,3,4,5,10$, and 15) is also compared in Fig. 4a. Both series present a very similar behaviour. Then, their respective correlation dimensions for every dimension $m$ have to be almost coincident. Figure $4 \mathrm{~b}$ depicts the evolution of $\mu(m)$, which reaches a stationary value, $\mu^{*}$, slightly exceeding 10.0 , for an embedding dimension $d_{\mathrm{E}}$ equal to 19 . This high $d_{\mathrm{E}}$ value is another sign of randomness. According to the meaning of $\mu^{*}$, a minimum number of 10 nonlinear equations is required to describe the 


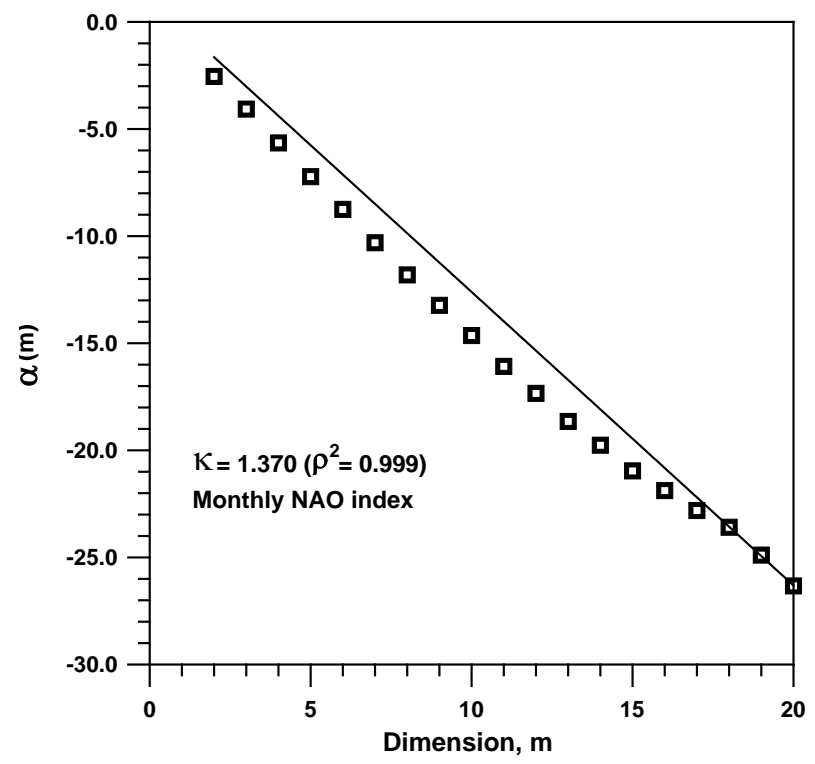

Fig. 5a. Linear evolution attributable to $m \kappa$ and its extrapolation up to $m$ equal to 2 (solid line). Open squares represent the relationship $\alpha(m)=\log _{\mathrm{e}}\left(A_{m}\right)-m \kappa$.

physical process, this high value enhancing its complexity. After the estimation of $\mu^{*}$, the Ruelle's condition (Ruelle, 1990)

$\mu^{*}<2 \log _{\mathrm{e}} \ell$

with $\ell$ equal to 2196 , the number of monthly NAO indices, suggests that $C(r)$ permits a reliable estimation of $\mu^{*}, d_{\mathrm{E}}$ and $\kappa$. Additional aspects regarding the confidence level of these estimations, especially for $\mu^{*}$, are discussed after the computation of Lyapunov exponents.

Figure 5a shows the evolution of $\alpha(m)$ with reconstruction dimension $m$. The Kolmogorov entropy is estimated by considering that the constraint $A_{m+1} \approx A_{m}$ is accomplished for $m \geq 18$, being obtained a value of 1.37 . The linear regression depicted in Fig. 5a corroborates this estimation, as the linear decrease for $m$ equal to 18, 19, and 20 differs from the general evolution of $\alpha(m)$. According to Eq. (9b), $\kappa$ would be underestimated by a 5-6\% if the linear regression was considered for $m$ ranging from 2 to 20 .

The last step of the reconstruction theorem is devoted to compute Lyapunov exponents, which quantify the sensitivity of the physical system to small perturbations on the starting conditions, thus being related to the predictive instability of monthly NAO indices. If $\delta \boldsymbol{Z}(m)_{0}$ is a small perturbation on the first reconstructed $\mathrm{m}$-dimensional vector, after the generation of infinite vectors $\boldsymbol{Z}(m)_{j}$, the largest Lyapunov exponent $\lambda_{\max }$ is defined as

$\lambda_{\max }=\lim _{j \rightarrow \infty} \frac{1}{j} \log _{\mathrm{e}}\left\|\frac{\delta \boldsymbol{Z}(m)_{j}}{\delta \boldsymbol{Z}(m)_{0}}\right\|$

the argument of the logarithm being the norm of the Jacobian

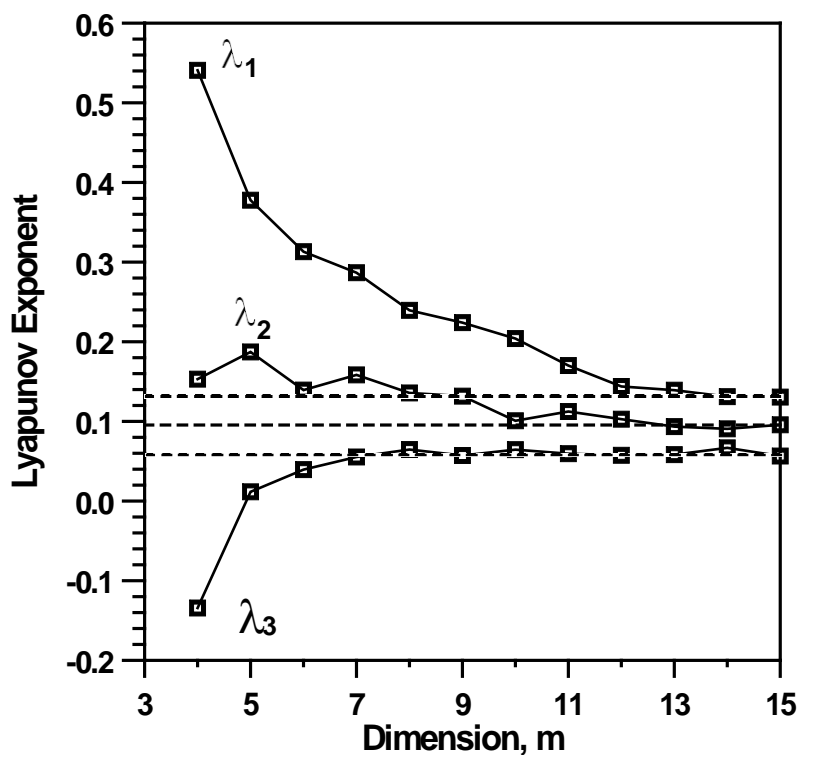

Fig. 5b. Evolution of the first three positive Lyapunov exponents with the reconstruction dimension $m$.

matrix. If $\lambda_{\max }$ is positive, the trajectory of the physical system diverges exponentially in the $\mathrm{m}$-dimensional space, being then characterised this dynamic system by a chaotic behaviour. Classic examples of these chaotic behaviours can be found analysing the van der Pol equation for spring-mass oscillation systems (Turcotte, 1997) or solutions of the Lorenz equations (Diks, 1999). Then, there is a strong dependence of perturbations on starting conditions and a long-term prediction is useless. The computation of the largest Lyapunov exponent can be generalised to the m Lyapunov exponents according to an iterative algorithm proposed by Eckmann et al. (1986) and Stoop and Meier (1988). In fact, $\lambda_{\max }$ is the highest positive Lyapunov exponent computed by the iterative algorithm. The evolution of the first three positive Lyapunov exponents when increasing dimension $m$ (Fig. 5b) leads to stationary positive values of $0.13,0.10$, and 0.06 for $\lambda_{1}, \lambda_{2}$ and $\lambda_{3}$, respectively, and reconstruction dimension $\mathrm{m}$ close to $d_{\mathrm{E}}$, certifying the predictive instability of monthly NAO indices. It has to be underlined that a single positive exponent is sufficient to generate predictive instability. Figure 6 illustrates the required number of iterations of the computational algorithm to reach a stationary value of the first three positive Lyapunov exponents for a reconstruction dimension $m$ equal to 15 . The first three Lyapunov exponents for the Gaussian white noise series are also determined. Table 1 lists and compares Lyapunov exponents derived for the monthly NAO indices and the Gaussian series. It is seen that differences are almost null. Consequently, the predictive instability and the chaotic behaviour of the NAO indices and the Gaussian series are very similar. 


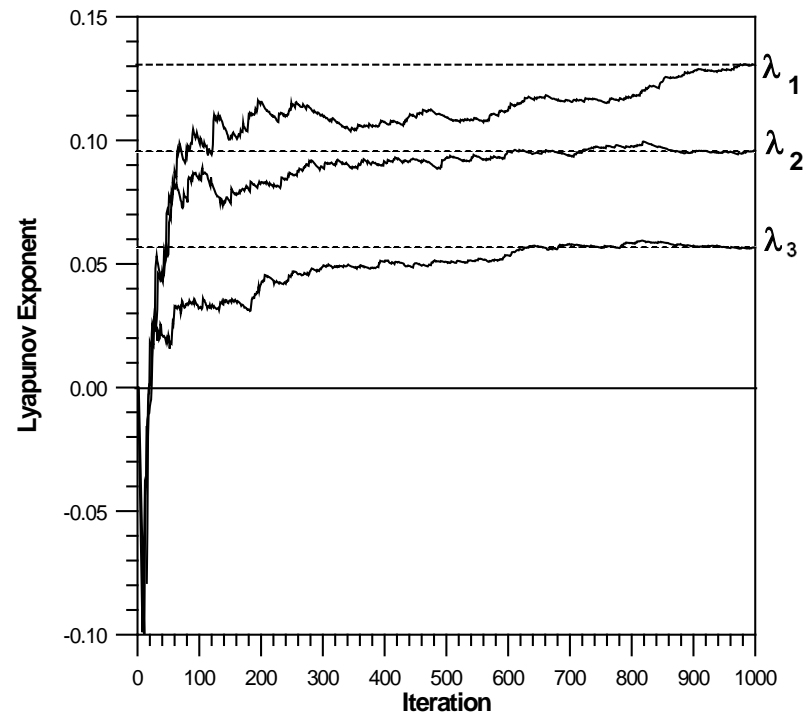

Fig. 6. Evolution of the first three positive Lyapunov exponents towards stationary values after 1000 iterations of the computational algorithm.

After the computation of the Lyapunov exponents, some aspects about a reliable estimation of $\mu^{*}, d_{\mathrm{E}}$ and $\kappa$ can be discussed. To assure convergence of the correlation dimension to a stationary value $\mu^{*}$, it should be also necessary the fulfilment of the relationship $d_{E}>2 \mu^{*}+1$, which implies exploring reconstruction dimensions $m$ above 20. Bearing in mind the evolution of $C(r)$ with the reconstruction dimension $\mathrm{m}$ in Fig. $4 \mathrm{a}$, it is difficult to reach so high dimensions $\mathrm{m}$ and to obtain confident estimations of $\mu(m)$. The increase in the effects of lacunarity and saturation of $C(r)$ to 1.0 notably reduce the range of $r$ for which $\mu(m)$ can be estimated. This range would be finally reduced to an inflection point. Consequently, after a revision of Fig. $4 \mathrm{~b}$, it could be assumed that a correlation dimension slightly exceeding 10.0 would be a lower (conservative) limit for the real value of $\mu^{*}$. Conceptually, the complexity of the nonlinear system of equations governing monthly NAO evolution is confirmed in spite of the uncertainty on $\mu^{*}$.

After a revision of Fig. 5a, it could be assumed that, although the evolution of $\alpha(m)$ with $m$ is only explored up to a reconstruction dimension equal to 20, the Kolmogorov entropy $\kappa$ should not be submitted to relevant uncertainties given that the linear evolution of $\alpha(m)$ for $m$ equalling to or exceeding 18 is quite clear. The embedding dimension $d_{\mathrm{E}}$ remains in some way undetermined (possibly underestimated) because a reliable stationary value of $\mu^{*}$ is not guaranteed. Nevertheless, the evolution of the first three Lyapunov exponents with the reconstruction dimension $\mathrm{m}$ (Fig. 5b) manifests that stationary values are reached for $m$ close to 15 . Consequently, the randomness of the monthly NAO indices is strongly suggested by the high value estimated for $d_{\mathrm{E}}$ (a minimum of 15), although higher embedding dimensions, as the
Table 1. Values of the first three Lyapunov exponents for monthly NAO index and for Gaussian noise.

\begin{tabular}{lccc}
\hline & $\lambda_{1}$ & $\lambda_{2}$ & $\lambda_{3}$ \\
\hline Monthly NAO index & 0.130 & 0.096 & 0.057 \\
Gaussian noise & 0.153 & 0.099 & 0.066 \\
\hline
\end{tabular}

Table 2. Values of parameters $H, d_{\mathrm{E}}, \mu^{*}, \kappa$ and $\lambda_{1}$ for monthly NAO index compared with a daily rainfall regime $\left({ }^{1}\right)$ and a dry spell distribution $\left({ }^{2}\right.$ ) (Martínez et al., 2007).

\begin{tabular}{lcrccc}
\hline & $H$ & $d_{\mathrm{E}}$ & $\mu^{*}$ & $\kappa$ & $\lambda_{1}$ \\
\hline Monthly NAO Index $^{c} \approx 0.5$ & $\geq 19^{\mathrm{a}}$ & 10.1 & 1.37 & 0.13 \\
Daily Rainfall $^{1}$ & $0.5-0.8$ & $15^{\mathrm{b}}$ & - & - & $0.15-0.35$ \\
Dry Spells $^{2}$ & $0.3-0.8$ & $15^{\mathrm{b}}$ & - & - & $0.15-0.40$ \\
\hline
\end{tabular}

${ }^{\text {a }}$ Estimated by searching for the stationary value of $\mu$.

${ }^{b}$ Estimated by searching for the stationary value of $\lambda_{1}$.

Kolmogorov entropy computation suggests, should not be discarded. As a summary, 10.0 could be a reasonable lower bound for the correlation dimension, and a Kolmogorov entropy of 1.37 a quite good estimation of the "loss of memory" of the dynamic system governing the monthly NAO indices. The embedding dimension should be at least equal to 15 , and possibly higher according to the evolution of $C(r)$ in Fig. 4a and the computation of $\kappa$.

\section{Discussion}

Some comparisons can be made between the present results and those derived for the daily rainfall and dry spell regimes of the Iberian Peninsula (Martínez et al., 2007). With respect to lacunarity, pairs of exponential laws for $L(1)$, similar to those of Eq. (5) for monthly NAO indices, were also obtained for the rainfall and dry spell regimes. A relevant novelty is that the empirical lacunarity of monthly NAO indices is well modelled by a random Cantor process, whereas it was not possible to fit the empirical lacunarities of the rainfall and dry spell regimes to theoretical curves derived from pure, random or clumped Cantor sets.

The results of the application of the rescaled analysis and the reconstruction theorem to the NAO indices, and to the daily rainfall and dry spell regimes of the Iberian Peninsula are summarised in Table 2 . The rescaled analysis of the annual and monthly NAO indices is characterised by a strong randomness $(H \approx 0.5)$. On the contrary, timepersistence is very likely for rainfall and dry spell regimes. Then, time trends should be a useful tool to predict drought episodes. However, using time trends for predicting future NAO indices would be questionable. The strong randomness 
of monthly NAO is also confirmed by several additional factors. First, the standardised monthly NAO indices follow a $N(0,1)$ distribution. Second, $L(1)$ behaves very similarly for monthly NAO and a Gaussian white noise series. Third, autocorrelation coefficients in Fig. $3 \mathrm{~b}$ (almost null) and Hurst exponents (very close to 0.5 ) are very similar for both series. The notable complexity and randomness of NAO index series is reflected by its embedding dimension $d_{\mathrm{E}}$, which exceeds that obtained for rainfall and dry spell regimes. Nevertheless, it should be taken into account that, in these last cases, $d_{\mathrm{E}}$ was deduced searching for stationary values of Lyapunov exponents. Possibly, $d_{\mathrm{E}}$ would be underestimated with respect to embedding dimensions deduced from stationary values of the correlation dimension. Predictive complexity of monthly NAO, and dry spell and rainfall regimes would be quite similar, as all these processes are governed by the same complex physical mechanism: the atmospheric dynamics. In spite of some shortcomings regarding estimation of $d_{\mathrm{E}}, \kappa$ and $\mu^{*}$, the Kolmogorov entropy underlines the notable loss of memory of NAO indices, which is in agreement with the absence of persistence and anti-persistence manifested by the Hurst exponent.

Although monthly NAO manifests notable random behaviour and loss of memory, the maximum Lyapunov exponent, $\lambda_{1}$, which is the main cause of predictive instability, does not differ considerably from those obtained for the daily rainfall and dry spell regimes. Nevertheless, it should be mentioned that for some threshold levels used to define dry spells, predictive instability is higher than for monthly NAO. An additional fact confirming NAO randomness is deduced by checking in Table 1 that the three first Lyapunov exponents derived for the monthly NAO indices and a Gaussian white noise series are very similar.

Several authors have proposed different predictive stochastic models for NAO index. Deterministic options (nonlinear systems of equations) should be discarded due to the high number of equations required, given by $\mu^{*}$, and the different signs suggesting a $N(0,1)$ distribution of standardised monthly NAO indices. A white noise uniform distribution might be an excessively simple way for describing the time evolution of the NAO index. Brownian and Markovian red-noise processes have been proposed by Collette and Ausloos (2004). NAO index prediction has also been attempted from other points of view (Mills, 2004), based on n-order autoregressive processes, but the same author recognizes that accurate predictions are difficult because cyclical and slowly changing components explain a low ratio of data variance in comparison with other irregular components. A long-range fractional integrated noise model instead of a random walk model has also been attempted by Stephenson et al. (2000), who assumed that natural climate variability could be a consequence of the aggregation of many stochastic weather processes. A similar deduction is obtained by Fernández et al. (2003) who propose that NAO series could be a realisation of many different stochastic processes. The same au- thors detect slight red-noise behaviour of the NAO index, in agreement with detrended fluctuation analysis by Caldeira et al. (2007), attributable to the fact that NAO would be driven by meteorological noise. Johansson (2007) establishes that forecasting at monthly and seasonal scales is not very efficient, excepting for winter season at short lead times. A similar conclusion is derived by Müller et al. (2005). Moreover, Fernández et al. (2003) and Caldeira et al. (2007) put the stress on the little predictive performance of the NAO index and the necessity for a deeper understanding of the underlying atmospheric dynamics. Conclusions of all these authors about NAO predictability would be in agreement with our main results: strong random behaviour (Hurst exponent very close to 0.5 ), predictive instability (positive Lyapunov exponents) and a complex physical mechanism (a high minimum number of nonlinear equations, at least 10 , according to $\mu^{*}$ ) manifesting a relevant loss of memory, in agreement with the concept of Kolmogorov entropy.

The relatively simple definition of the NAO index contrasts with the complex patterns of the atmospheric dynamics and fractal properties of NAO itself. According to Wallace (2000), NAO would be integrated on a larger scale atmospheric fluctuation, the Northern Annular mode or Artic Oscillation (Stephenson et al., 2000; Ambaum et al., 2001) and the NAO index should be assumed as a partial insight into a complex atmospheric dynamics. Randomness and predictive instability could be then partially attributable to the complexity of the physical process governing NAO, but could be also the consequence of a not very appropriate characterisation of the atmospheric dynamics by the NAO index. Some authors have even suggested sea level pressure measurements in new locations and/or time scales modifying nowadays monthly NAO definition (Collette and Ausloos, 2004).

\section{Conclusions}

The physical process governing monthly NAO indices can be qualified as a dissipative chaotic dynamic system describing asymptotic stable aperiodic trajectories around some points, known as strange attractors, in the m-dimensional reconstructed space and with predictions very sensitive to initial conditions. The most relevant signs of complex predictability would be:

a) An evident random behaviour, suggested by random Cantor sets reproducing empirical lacunarities, Hurst exponents close to 0.5 (at annual and monthly scales) and high embedding dimensions.

b) Several signs suggesting behaviour of the monthly NAO index quite similar to a Gaussian white-noise distribution.

c) A high minimum number of nonlinear equations required to quantify the physical process (the atmospheric dynamics) governing monthly NAO.

d) Positive Lyapunov exponents generating predictive instability and chaotic behaviour. 
In summary, our results indicate that NAO prediction would require high dimensional stochastic models, and that the prediction would be significantly limited by errors in initial conditions. As random Cantor models reproduce satisfactorily monthly NAO lacunarities for a wide range of threshold values, these random models, together with the Gaussian distribution, should be considered for predictive strategies of monthly NAO indices. In addition, it should not be discarded that shortcomings affecting predictability could partially be artefacts of the quite simple definition of NAO index.

Acknowledgements. The authors are indebted to the editor and reviewers for their useful suggestions and comments.

Edited by: O. Talagrand

Reviewed by: M. Ausloos and three other anonymous referees

\section{References}

Ambaum, M. H. P., Hoskins, B. J., and Stephenson, D. B.: Arctic oscillation or North Atlantic oscillation?, J. Climate, 14, 34953507, 2001.

Cohen, A. and Procaccia, I.: Estimation of Kolmogorv entropy from time signals of dissipative and conservative dynamical systems, Phys. Rev., A, 31, 1872-1882, 1983.

Bera Anil, K. and Jarque, C. M.: Efficient tests for normality, homoscedasticity and serial independence of regression residuals, Econ. Lett., 7(4), 313-318, 1981.

Caldeira, R., Fernández, I., and Pacheco, J. M.: On NAO's predictability through the DFA method, Meteorol. Atmos. Phys., 96, 221-227, 2007.

Collette, C. and Ausloos, M.: Scaling analysing and evolution equation of the North Atlantic oscillation index fluctuations, Int. J. Mod. Phys. C, 15(10), 1353-1366, 2004.

D’Agostino, R. B., Belanger, A., and D'Agostino Jr., R. B.: A suggestion for using powerful and informative tests of normality, Am. Stat., 44(4), 316-321, 1990.

Diks, C.: Nonlinear Time Series Analysis. Methods and Applications. Nonlinear Time Series and Chaos, 4. World Scientific, London, 209 pp., 1999.

Eckmann, J. P., Oliffson, S., Ruelle, D., and Cilliberto, S.: Lyapunov exponents from time series, Phys. Rev., A, 34(6), 49714979, 1986.

Feldstein, S. B.: The timescale, power spectra and climate noise properties of teleconnection patterns, J. Climate, 13, 4413-4440, 2000.

Fernández, I., Hernández, C. N., and Pacheco, J. M.: Is the North Atlantic Oscillation just a pink noise?, Physica A, 323, 705-714, 2003.

Grassberger, P. and Procaccia, I.: Characterization of strange attractors, Phys. Rev. Lett., 50, 346-349, 1983 a.

Grassberger, P. and Procaccia, I.: Estimation of the Kolmogorov entropy from a chaotic signal, Phys. Rev., A, 28, 2591, doi:10.1103/PhysRevA.28.2591, 1983 b.

Hurrell, J. W., Kuschnir, Y., and Visbeck, M.: The North Atlantic oscillation, Science, 291, 603-605, 2001.
Johansson, Å.: Prediction skill of the NAO and PNA from daily to seasonal time scales, J. Climate, 20, 1957-1975, 2007.

Jones, P. D., Jonsson, T., and Wheeler, D.: Extension to the North Atlantic Oscillation using early instrumental pressure observations from Gibraltar and South-West Iceland, Int. J. Climatol., 17, 1433-1450, 1997.

Korvin, G.: Fractals Models in the Earth Sciences, Elsevier, Amsterdam, 396 pp., 1992.

Krichak, S. O. and Alpert, P.: Signatures of the NAO in the atmospheric circulation during wet winter months over the Mediterranean region, Theor. Appl. Climatol., 82, 27-39, 2005.

Lomnitz, C.: Fundamentals of Earthquake prediction, Wiley, New York, 1994.

Mandelbrot, B. B.: The Fractal Geometry of Nature, Freeman, San Francisco, 1982.

Marshall, J., Kushnir, Y., Battisti, D., Chang, P., Czaja, A., Dickson, R., Hurrell, J., McCartney, M., Saravanan, R., and Visbeck, M.: North Atlantic climate variability: phenomena, impacts and mechanisms, Int. J. Climatol., 21, 1863-1898, 2001.

Martín, M. L., Luna, M. Y., Morata, A., and Valero, F.: North Atlantic teleconnections patterns of low-frequency variability and their links with springtime precipitation in the Western Mediterranean, Int. J. Climatol., 24, 213-230, 2004.

Martínez, M.D., Lana, X., Burgueño, A., and Serra, C.: Lacunarity, predictability and predictive instability of the daily pluviometric regime in the Iberian Peninsula, Nonlin. Processes Geophys., 14, 109-121, 2007, http://www.nonlin-processes-geophys.net/14/109/2007/.

Mills, T. C.: Is the North Atlantic oscillation a random walk? A comment with further results, Int. J. Climatol., 24, 377-383, 2004.

Müller, W. A., Appenzeller, C., and Schär, C.: Probabilistic seasonal prediction of the winter North Atlantic Oscillation and its impact on near surface temperature, Clim. Dynam., 24, 213-226, 2005.

Muñoz-Díaz, D. and Rodrigo, F. S.: Impacts of the North Atlantic Oscillation on the probability of dry and wet winters in Spain, Clim. Res., 27, 33-43, 2004.

Ruelle, D.: Deterministic Chaos: The Science and the Fiction, Proc. R. Soc. Lon. Ser.-A, 427, 241-248, 1990.

Stephenson, D. B., Pavan, V., and Bojariu, R.: Is the North Atlantic oscillation a random walk?, Int. J. Climatol., 20, 1-18, 2000.

Stoop, F. and Meier, P. F.: Evaluation of Lyapunov exponents and scaling functions from time series, J. Opt. Soc. Am., B, 5, 10371045, 1988.

Theiler, J.: Lacunarity in a best estimation of cluster dimension, Phys. Lett. A, 133, 195-200, 1988.

Trigo, R. M., Osborn, T. J., and Corte-Real, J. M.: The North Atlantic Oscillation influence on Europe: climate impacts and associated physical mechanisms, Clim. Res., 20, 9-17, 2002.

Turcotte, D. L.: Fractal and Chaos in Geology and Geophysics, 2nd edn., Cambridge University Press, 398 pp., 1997.

Ulbrich, U. and Christoph, M.: A shift of the NAO and increasing storm track activity over Europe due to anthropogenic greenhouse gas forcing, Clim. Dynam., 15, 551-559, 1999.

Wallace, J. M.: North Atlantic oscillation/annular mode: Two paradigms - one phenomenon, Q. J. Roy. Meteor. Soc., 126, 791806, 2000. 